\title{
Investigando os padrões dos alunos em ambientes massivos
}

\author{
Ana Carla A. Holanda ${ }^{1,2}$, Patrícia, C. A. R. Tedesco ${ }^{1}$, Rodrigo, S. Souza ${ }^{2}$ \\ ${ }^{1}$ Universidade Federal de Pernambuco (UFPE) \\ Caixa Postal 50.540-740 - Recife - PE - Brasil \\ ${ }^{2}$ Instituto Federal do Acre (IFAC) \\ Caixa Postal 69.900-640 - Rio Branco - AC - Brasil \\ \{acah@cin.ufpe.br, pcart@cin.ufpe.br, rodrigo.souza@ifac.edu.br\}
}

\begin{abstract}
The popularity of MOOCs (Massive Open Online Course) has limited time and space barriers in traditional education and has become an important source of access to quality resources. However, the increase in the number of students on the platform with different levels of knowledge, goals and needs hinders interaction in the environment, due to different contexts where students are inserted. In this way, this article aimed to examine the patterns of student activities to identify actions that can be taken to make the environment more interactive, thus encouraging shared learning and built from different perspectives and perspectives.
\end{abstract}

\section{Resumo}

A popularidade dos MOOCs (Curso Online Aberto Massivo) tem limitado as barreiras de tempo e espaço na educação tradicional e tem se tornado uma importante fonte de acesso a recursos de qualidade. Entretanto, o aumento do número de alunos na plataforma com diferentes níveis de conhecimentos, metas e necessidades dificulta a interação no ambiente, devido a diferentes contextos onde os alunos estão inseridos. Desta forma, este artigo teve o objetivo de examinar os padrões de atividades dos alunos para identificar ações que podem ser realizadas para tornar o ambiente mais interativo, incentivando assim, a aprendizagem compartilhada e construída a partir de diferentes olhares $e$ perspectivas.

\section{Introdução}

Massive Open Online Courses (MOOC) atraem participantes com uma multiplicidade de conhecimentos e com uma variedade de motivações e propósitos. Segundo Antonaci et al. (2017) com o objetivo de trazer e democratizar a educação para todos, MOOCs começaram a crescer nos últimos anos, por hipoteticamente, trazer educação para todos sem custo. Holanda e Tedesco (2017) complementam ao afirmar que MOOCs proporcionam uma grande diversidade de conteúdos de qualidade. Apesar desse potencial, estudos do MOOC destacaram várias limitações, como a falta de engajamento dos usuários (ZHENG, 2016) e sua alta evasão (WAKS, 2016) e, consequentemente, baixas taxas de conclusão (ZHOU, 2016).

Entretanto, é importante destacar que cursos massivos não devem ser vistos como cursos tradicionais, tendo em vista que ambientes massivos podem atingir potencialmente 
VIII Congresso Brasileiro de Informática na Educação (CBIE 2019)

Anais do XXX Simpósio Brasileiro de Informática na Educação (SBIE 2019)

um grande número de pessoas, sendo necessário lidar com uma enorme variedade de necessidades e objetivos. Holanda e Tedesco (2017) comentam que um dos desafios encontrados na construção e condução destes cursos é a dificuldade em mapear os perfis dos alunos baseando-se em seu comportamento e interação.

Assim, esta pesquisa teve como objetivo examinar os padrões de atividades dos alunos, incluindo informações demográficas e objetivos pessoais enquanto inscritos no MOOC. Os resultados incitam alternativas para apoiar estudantes neste ambiente, através da aplicação de ferramentas e estratégias que potencializem o processo de aprendizagem.

O artigo está organizado da seguinte forma: a fundamentação teórica é discutida na Seção 2; a metodologia é descrita na Seção 3; os resultados e as discussões das análises são apresentados na Seção 4. E, finalmente, as conclusões e trabalhos futuros são explorados na Seção 5.

\section{FUNDAMENTAÇÃO TEÓRICA}

A discussão sobre MOOC e seu papel e impacto continuam a ser intensos, e é notável quanta disputa ainda está em curso em torno de questões muito básicas. A palavra massiva refere-se ao grande número de alunos que estão simultaneamente inscritos, de centenas a milhares de participantes. A massividade é um desafio, pois identificar os diferentes perfis e objetivos dos alunos e adaptar o ambiente a essas necessidades pode melhorar o engajamento e a motivação dos participantes, proporcionando um compartilhamento de ideias e, assim, a possibilidade de potencializar a aprendizagem.

Downes (2012) explica que o objetivo do MOOC não é transmitir conteúdo e sim fazer com que as pessoas troquem ideias e criem uma comunidade de aprendizado conectivista. A partir de então, várias universidades disponibilizaram cursos massivos como uma alternativa para gerar uma maior qualificação e aprendizagem para uma ampla quantidade de pessoas através da Internet. Entretanto, apesar da aceitação generalizada destes cursos como uma oportunidade para transformar as práticas educacionais, ainda existem muitos desafios a serem superados para garantirem aspectos colaborativos e de interação nesses ambientes.

\subsection{Valor Educacional de MOOCs}

Waks (2016) faz uma análise do valor educacional dos MOOCs verificando de que forma eles podem beneficiar a aprendizagem dos alunos. Então ele considera que MOOCs tem os seguintes valores:

- Valor instrumental - MOOCs são um novo tipo de instrumento educacional, então pode-se considerar seu valor como instrumento para alcançar fins educacionais.

- Valor técnico - segundo o autor, o valor técnico é fundamental para educação e os MOOCs são considerados meios de transmissão de conhecimento que automatizam e ampliam as possibilidades de acesso ao conhecimento, ajudando os alunos a melhorarem o seu nível intelectual, pois possibilita o crescimento de habilidades e técnicas que constroem o conhecimento.

- Valor hedônico - é o valor do prazer ou satisfação. Na educação, o valor hedônico acompanha muitos tipos de atividades de aprendizagem, por exemplo, palestras fascinantes, professores divertidos, a possibilidade de 
VIII Congresso Brasileiro de Informática na Educação (CBIE 2019)

Anais do XXX Simpósio Brasileiro de Informática na Educação (SBIE 2019)

guiar o seu próprio caminho, o envolvimento ativo de atividades e debates que promove o engajamento, levando em consideração a experiência consumada que trazem satisfação ao aluno e professor. Estas características podem ser evidenciadas em MOOCs, desde que o ambiente seja planejado para potencializar este tipo de sensação nos alunos.

- Valor de uso - o valor educativo de uma experiência reside nas duas características de interação e continuidade. Primeiro, a experiência tem que envolver os alunos; eles têm que interagir com materiais de aprendizagem, encontrar neles algo relacionado aos seus objetivos e buscarem oportunidades para alcançá-los. E depois, a experiência deve ser contínua com a vida do aluno, sendo útil para o mesmo, que recorrer ao aprendizado prévio e contribui para o acúmulo de capacidades e experiências. É importante ter em mente que tudo que é benéfico para o aluno tem valor de uso, pois na avaliação educacional o valor é central.

Entretanto, apesar das perspectivas e crescimento dos MOOCs estes passaram a receber críticas quanto ao seu uso. Stein (2013) comenta que as críticas se referem ao fato de que os cursos usam a mesma pedagogia ultrapassada dos cursos presenciais e não estão trazendo inovação para o ambiente de aprendizagem, visto que apenas estendem a sala de aula em um ambiente online sem considerar as necessidades de cada aluno inscrito no curso.

Além disso, também é discutido que os (1) MOOCs têm baixas taxas de conclusão, (2) não podem substituir funções de ensino essenciais e; (3) estão isolando, enquanto o aprendizado é social. (WAKS, 2016). Zhou (2016) aponta também (4) as barreiras linguísticas, por desfavorecer a aprendizagem àqueles que não tem conhecimento na língua. As características individuais e os (5) diferentes estilos de aprendizagem dos alunos pode ser um entrave ao modelo educacional de MOOCs (THAIPISUTIKUL e TUAROB, 2017). Além disso, os (6) diferentes padrões de comportamentos dos alunos dificultam o planejamento pedagógico dos cursos. Zheng (2016) comenta que é uma tarefa complexa (7) incentivar a participação e engajamento dos estudantes de MOOCs.

\section{METODOLOGIA}

\subsection{Problema de pesquisa}

Esta pesquisa teve como objetivo examinar os padrões de atividades dos alunos, incluindo informações demográficas e motivação para compreender a forma como deve-se planejar as aulas e disponibilizar ferramentas em um ambiente massivo. Assim, o problema de pesquisa deste artigo foi: "Quais as práticas colaborativas realizadas no ambiente MOOC?"

\subsection{Design da pesquisa}

Para responder a esta pergunta foi utilizado o estudo de caso como método de pesquisa, pois, segundo Pimentel (2011) é usado para avaliar se a teoria se verifica na prática e é adequado quando se busca o realismo contextualizado em vez da precisão laboratorial livre de contexto. O método estudo de caso deve ser escolhido quando se deseja realizar uma pesquisa que envolva questões comportamentais e sociais complexas decorrentes de múltiplas interações, cuja investigação pode estar muito além da capacidade de um único experimento. 
VIII Congresso Brasileiro de Informática na Educação (CBIE 2019)

Anais do XXX Simpósio Brasileiro de Informática na Educação (SBIE 2019)

Será usada uma abordagem exploratória, pois Pimentel (2011) afirma que num estudo de caso exploratório, o objetivo é explorar as situações em que o fenômeno ocorre. É uma abordagem útil para levantar problemas, identificar variáveis relacionadas ao fenômeno, investigar possíveis causas e consequências, e para elaborar algumas proposições.

\subsection{Unidade de análise}

A unidade de análise do estudo são alunos inscritos curso MOOC de HTML 5 Introdução ao Front-end do Instituto Federal do Acre - IFAC. A partir dos dados da interação do aluno neste curso foi possível identificar quais os padrões de comportamentos dos participantes.

\subsection{Coleta de Dados}

Os dados para esta análise foram coletados a partir de dados constantes no Banco de Dados do curso acima descrito. Além disso, foram utilizados os seguintes instrumentos para coleta de dados:

1 - Um questionário para coletar dados demográficos e motivações dos alunos;

2 - Realização de um teste inicial, a partir da inscrição no curso para quantificar o conhecimento inicial do aluno antes da realização do curso;

3 - Realização de um teste final após a realização do curso.

A Figura 1, abaixo, mostra o design da pesquisa proposta:

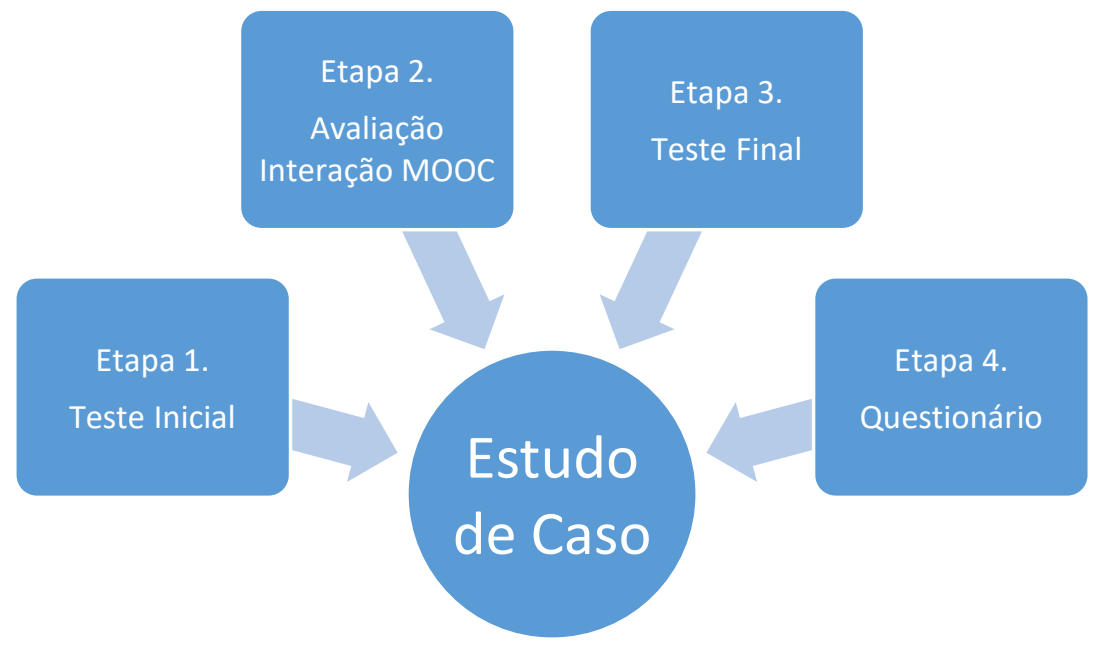

Figura 1. Design da pesquisa

\section{RESULTADOS}

Foi realizado um estudo de caso na plataforma MOOC do Instituto Federal do Acre ${ }^{1}$ no curso de HTML 5 - Introdução ao Front-end com 265 alunos inscritos no período de abril a maio de 2019. O curso foi dividido em 12 aulas, onde foram disponibilizados vários vídeos, atividades e materiais complementares. Não há nenhuma restrição em acessar aulas ou materiais em ordens não-sequenciais, pois a realização das atividades não é um critério obrigatório para dar sequência ao curso.

1 Disponível em: http://cursos.ifac.edu.br/ 
No espaço é disponibilizado apenas o fórum como ferramenta de colaboração no ambiente, não tendo nenhum meio de ferramenta síncrona no ambiente, o que dificulta a colaboração entre os alunos no curso. Também não há uma identificação dos colegas de curso por não está disponível a lista de alunos inscritos. Estas características dificultam a promoção da aprendizagem colaborativa dentro do ambiente, tão necessária para cursos MOOC, por compreender que o processo de aprendizagem em rede depende dos participantes para acontecer. Devido a diversidade de alunos, também há uma pluralidade de níveis de conhecimento e não há nenhum meio de avaliar o conhecimento individual ao iniciar o curso, o que pode dificultar a sequência do aluno no decorrer do curso.

\subsection{Aplicação de questionário}

Foi aplicado um questionário ${ }^{2}$ para coletar dados demográficos e motivacionais dos alunos. Através deste questionário também foi possível mensurar os pontos positivos e negativos da plataforma sob o olhar do aluno.

O questionário foi dividido em 6 partes: a primeira parte teve a intenção de coletar os dados demográficos dos alunos; a $2^{a}$ parte coletou dados sobre a estrutura do curso; a $3^{\mathrm{a}}$ parte avaliou os dados relacionados à interação do ponto de vista do aluno; a $4^{\mathrm{a}}$ etapa identificou aspectos gráficos do curso; a $5^{\text {a }}$ etapa identificou os dados de usabilidade do ambiente e; por fim, a $6^{\text {a }}$ etapa que fez uma avaliação das estratégias de aprendizagem do ambiente.

Ao analisar os dados do questionário percebe-se que os alunos estão distribuídos com maior concentração na região norte, conforme visto na Figura 2, abaixo:

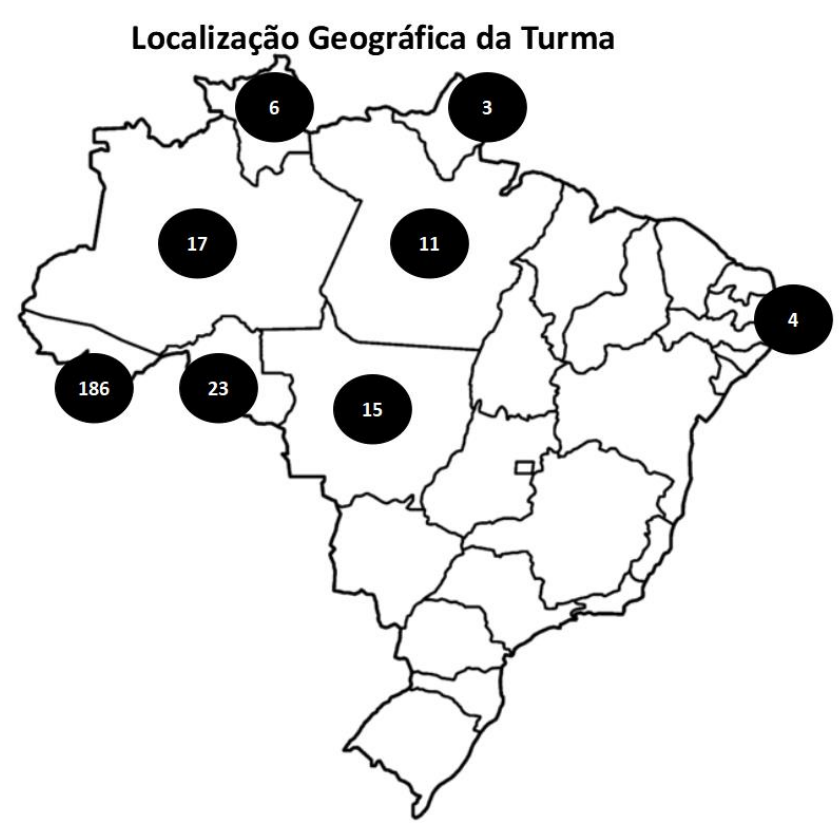

Figura 2. Localização Geográfica da turma

Quanto ao sexo e idade dos alunos matriculados percebe-se a seguinte distribuição:

2 Disponivel em: https://docs.google.com/forms/d/1ypVsjwrQ2R5OWbXNKu5DBcUt0kswOPyF2fnOtg3JwV4/prefill 
VIII Congresso Brasileiro de Informática na Educação (CBIE 2019)

Anais do XXX Simpósio Brasileiro de Informática na Educação (SBIE 2019)

Sexo e idade dos alunos inscritos

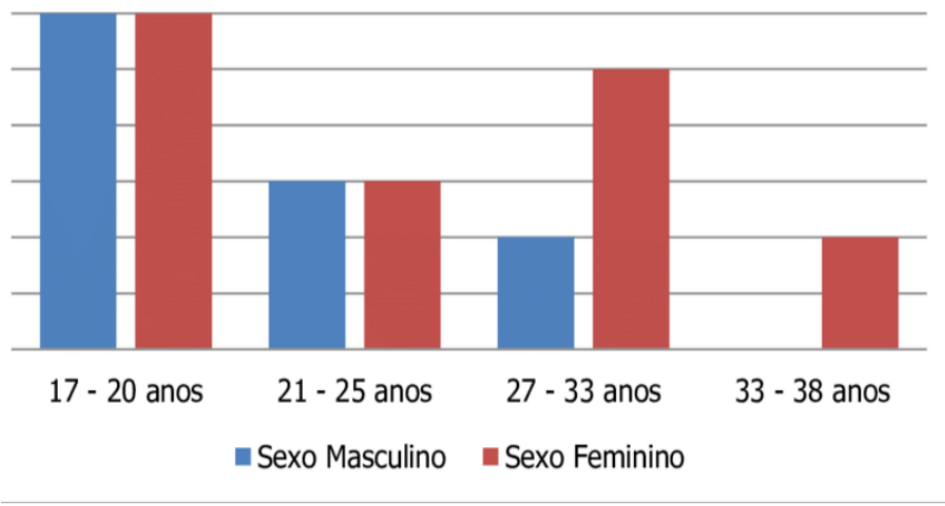

Figura 3. Sexo e idade dos alunos

Para compreender melhor como os alunos interagem no ambiente foram observadas as seguintes ações no ambiente: frequência de acesso ao ambiente, posts (lidos e escritos) nos fóruns, visualizações em vídeos, atividades realizadas e emissão da certificação.

Ao analisar a frequência dos logs no ambiente, percebe-se que eles vêm diminuindo com o passar do tempo. Assim, no início observa-se a maior quantidade de acessos na plataforma e o maior tempo de permanência no ambiente. Ao longo do curso essa frequência de acesso e permanência vem diminuindo consideravelmente, como pode ser visto na Figura 4, abaixo:

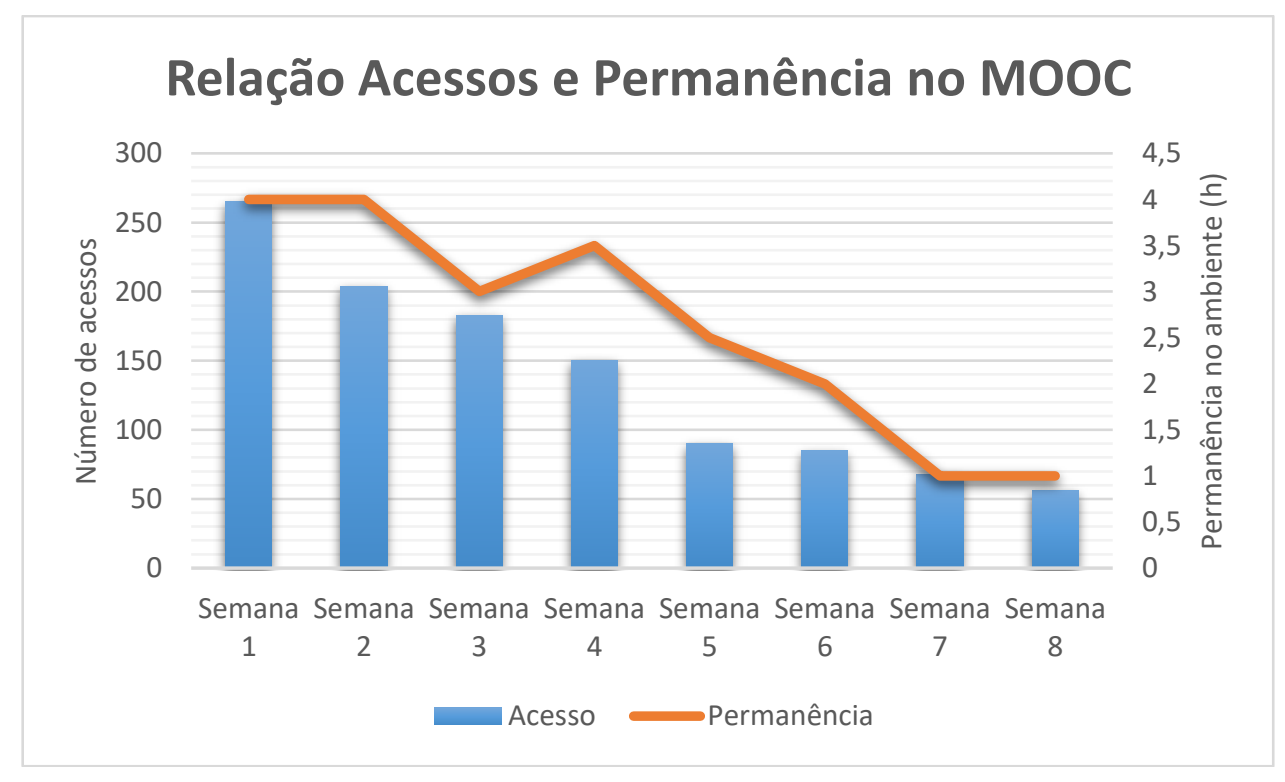

Figura 4. Relação entre acessos e permanência no ambiente 
VIII Congresso Brasileiro de Informática na Educação (CBIE 2019)

Anais do XXX Simpósio Brasileiro de Informática na Educação (SBIE 2019)

Estes achados permitem dar uma pista de que à medida que o tempo passa, os alunos vão perdendo a motivação na continuidade do curso, o que aponta um alerta sobre as possíveis causas dessa falta de engajamento no decorrer do curso.

$\mathrm{Na}$ análise dos fóruns, observa-se que o mesmo não é um canal frequente de comunicação entres os inscritos no curso, como pode ser visto na Figura 5. De uma forma geral, houve pouca interação entre os alunos no decorrer das semanas e essa participação foi gradativamente diminuindo com o andamento do curso. Buscando compreender as possíveis causas dessa inatividade no canal de comunicação, as respostas constantes no questionário puderam tentar justificar essa atitude no ambiente. Muitos alunos em seus relatos citaram a sensação de isolamento no ambiente, tendo em vista que não tinham conhecimento de seus colegas de curso, pois em local nenhum no ambiente havia o registro dos inscritos no curso, ou um momento de apresentação entre eles.

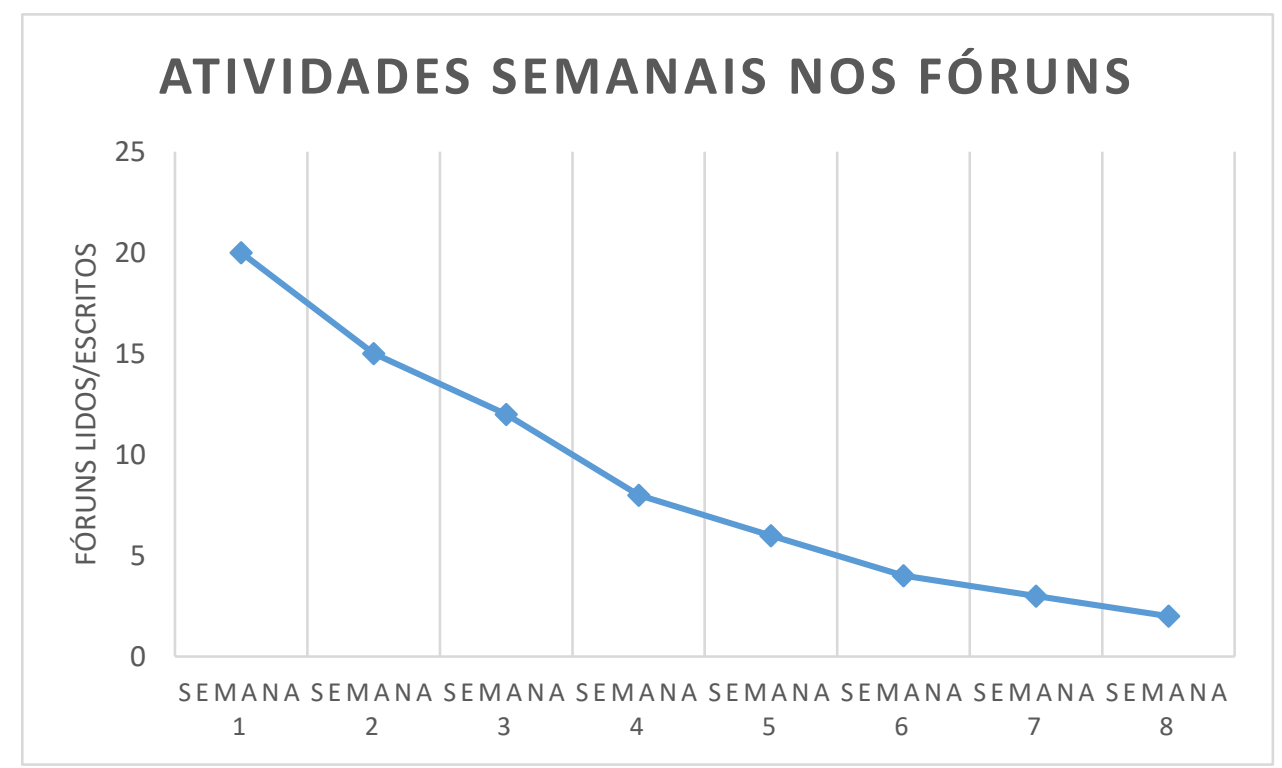

Figura 5. Atividades semanais nos fóruns

Em relação aos vídeos, percebe-se que o tempo médio de visualização dos vídeos é de 5 minutos, ou seja, é importante produzir vídeos com duração média observando esta característica. Observa-se também que na primeira semana, os alunos visualizam com maior tempo os vídeos da primeira aula. As demais aulas eles assistem o primeiro minuto e depois deixam de assisti-lo. Essa informação nos dá indícios de que os alunos estão interessados em visualizar os vídeos para saber o que vai ser abordado no decorrer das aulas. Nas semanas seguintes, percebe-se que o tempo médio para assistir os demais vídeos aumentam e os alunos tendem a finalizá-lo.

Quanto à realização das atividades realizadas, observa-se uma relação direta com os vídeos assistidos. É importante destacar que ao final de cada vídeo são disponibilizadas as atividades, o que pode explicar essa relação direta. Para realizar as atividades, o aluno pode fazê-lo sem um limite de tentativas. O sistema está configurado para registrar a nota mais alta das pontuações dos alunos. Do ponto de vista pedagógico, essa estratégia possibilita compreender melhor os padrões de interação dos alunos, e do ponto de vista psicológico, espera-se que tal sistema reduza o estresse e, portanto, faz com que os alunos se comportem de maneira mais confortável. A Figura 6, mostra como ocorre essa relação. 
VIII Congresso Brasileiro de Informática na Educação (CBIE 2019)

Anais do XXX Simpósio Brasileiro de Informática na Educação (SBIE 2019)

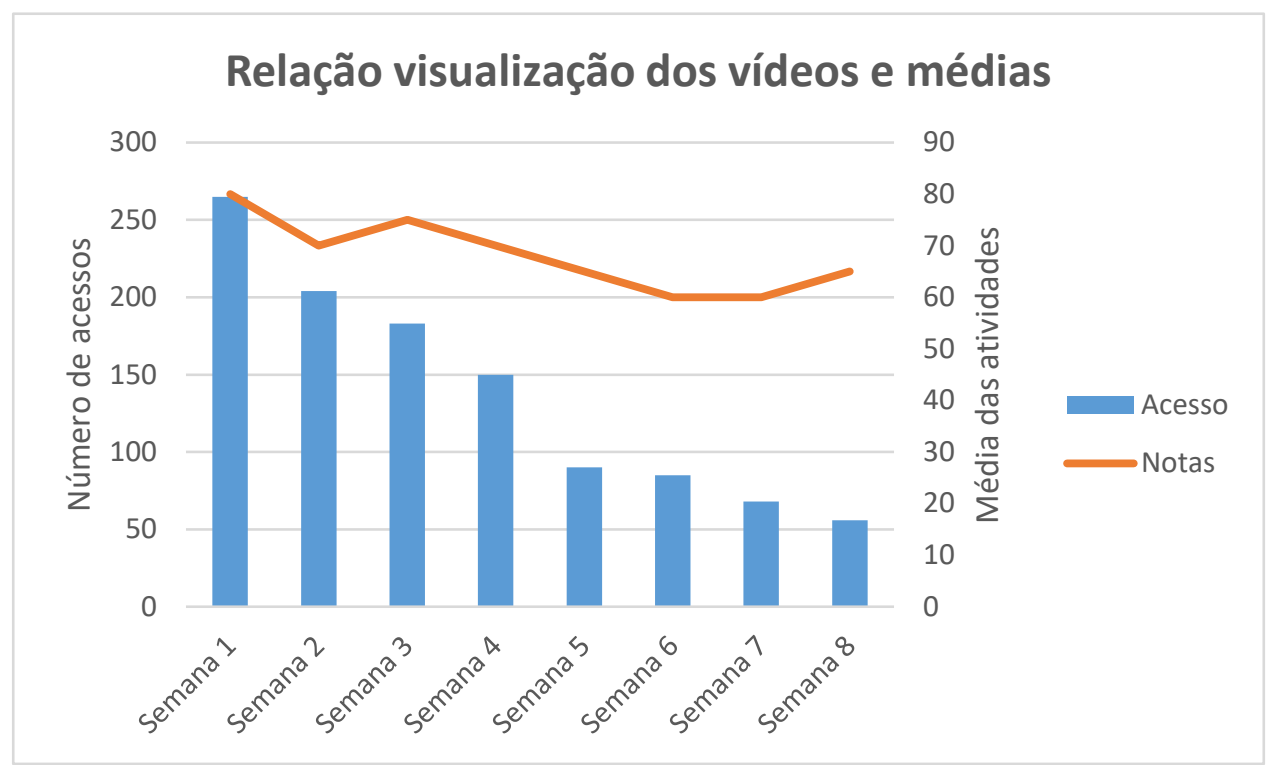

Figura 6. Relação vídeos e médias das atividades

Ao analisar as respostas dos questionários no que se refere às informações do curso percebeu-se que os alunos tiveram acesso às informações essenciais sobre a evolução do curso e os mesmos também consideraram o material de boa qualidade. Quanto à abordagem utilizada durante as aulas (vídeos, atividades e fóruns) a maioria considerou razoável e citaram a sensação de isolamento devido à falta de um ambiente mais participativo entre os envolvidos no curso. Também foi citado o fato de que não tiveram nenhum tipo de lembrete ou mensagem que pudesse motivá-los a continuarem a realizar o curso.

Outra informação que pôde ser identificada foi a necessidade do ambiente possibilitar a colaboração entre os alunos por meio da discussão sobre os temas que estavam sendo abordados nas atividades. Cerca de $83 \%$ dos alunos responderam positivamente a essa alternativa. Os alunos puderam justificar a sua resposta, então, comentários como "Acho relevante, pois assim posso trocar ideias com outras pessoas", "Se eu conversar com outro aluno não vou ter vergonha em mostrar a minha dúvida, pois nem todos irão visualizar isso no ambiente”, "É legal, mas tem de ser alguém que possa contribuir comigo", "Acho que conversando com outra pessoa a gente aprende mais", "Eu acho que quando tiro dúvidas de alguém eu aprendo muito mais" nos chamaram atenção. Estes relatos nos mostram que a colaboração pode beneficiar a aprendizagem em vários aspectos: tanto do ponto de vista das pessoas que têm receio em expor publicamente a sua dúvida, quanto do ponto de vista daqueles que acreditam que podem melhorar o seu conhecimento a partir do momento que auxiliam alguém.

\subsection{Testes de habilidades}

A partir da inscrição do aluno no curso foi disponibilizado um teste inicial para identificar as habilidades prévias de cada aluno. $\mathrm{O}$ teste inicial ${ }^{3}$ foi elaborado levando em consideração conceitos básicos sobre HTML e visava mensurar as habilidades iniciais dos alunos antes da realização do curso.

\footnotetext{
3 Disponível em: https://drive.google.com/open?id=1aPQm0tkHi9-MtN7tEKrQtyxasX6xj-_g
} 
VIII Congresso Brasileiro de Informática na Educação (CBIE 2019)

Anais do XXX Simpósio Brasileiro de Informática na Educação (SBIE 2019)

Ao final do curso também foi disponibilizado um teste final ${ }^{4}$ para mensurar o conhecimento adquirido, após da realização do curso. A partir dessas informações foi possível fazer uma comparação dos conhecimentos inicial e final para analisar se o curso trouxe aprendizagem significativa para os alunos.

Analisando os resultados dos dois testes, visto na Figura 7, percebe-se que a curva de aprendizagem ao final do curso aumentou de forma muito moderada. Em muitos casos, percebe-se que as notas finais foram menores das iniciais. Isso pode ser explicado pelo fato da falta de motivação e engajamento no curso e a falta de feedback e colaboração entre os envolvidos no curso.

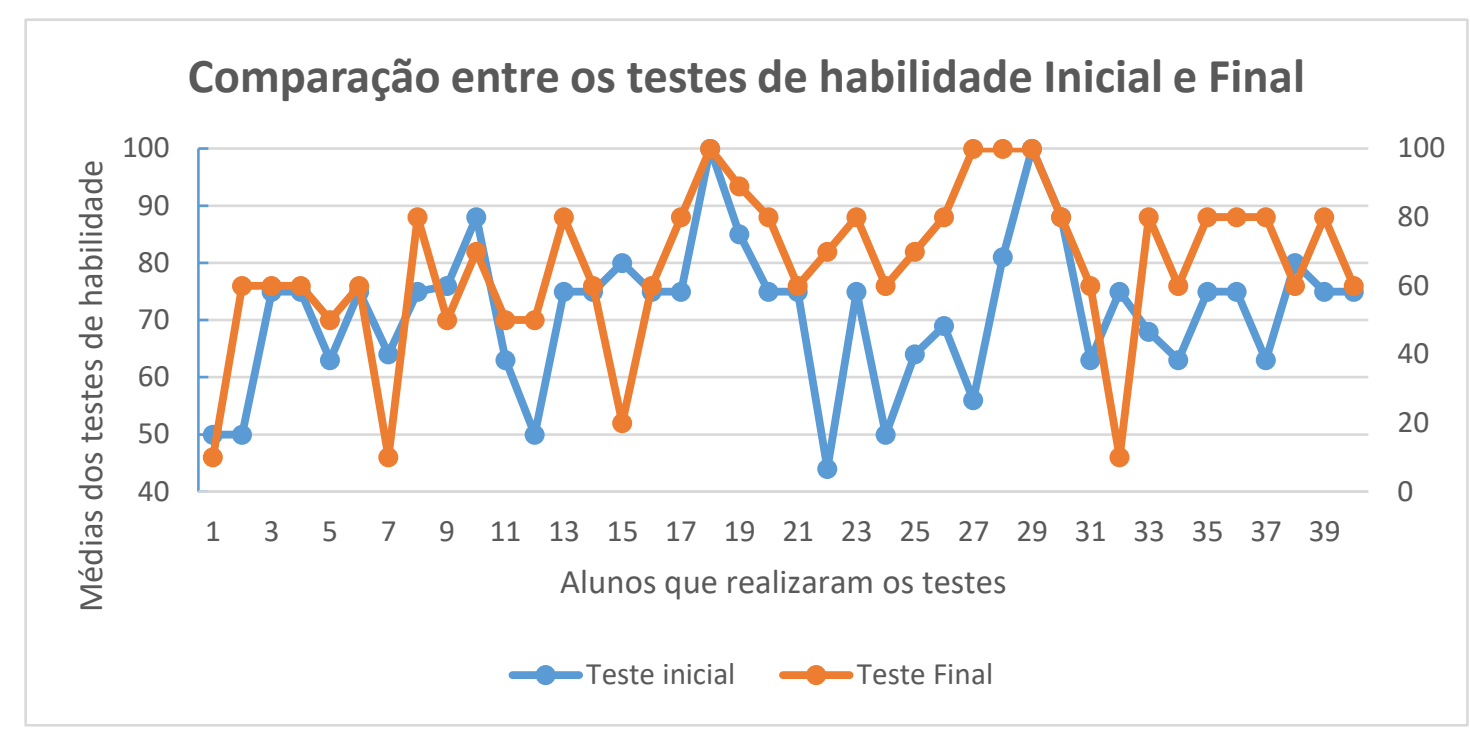

Figura 7. Comparação entre os testes de habilidade

De uma forma geral foi identificado que o formato do MOOC através de vídeos, atividades e fóruns é uma abordagem de disponibilizar aulas com materiais de qualidade (segundo o entendimento dos alunos matriculados no curso), mas para a construção de um conhecimento ativo, opções de suporte e colaboração são necessários, tendo em vista que durante o andamento do curso, a interação dos alunos no ambiente diminuiu consideravelmente.

Em relação ao único canal de comunicação disponível, a sua utilização se mostrou muito tímida, desde o início do curso e houveram poucas interações durante todo o curso. Ao analisar as respostas do questionário, percebe-se que muitos alunos se sentiram isolados e desmotivados devido a atual estrutura e design da plataforma.

\section{CONSIDERAÇÕES FINAIS}

A acelerada expansão e disseminação das tecnologias digitais, tornou os MOOCs numa modalidade de distribuição massiva do conhecimento, proporcionando acesso a uma educação mais aberta e flexível. Com repercussões diretas nas práticas pedagógicas, assume-se a necessidade de refletir sobre os procedimentos inerentes à sua construção e produção e de analisar a forma como estes novos modelos de ensino favorecem as experiências de aprendizagem colaborativa em um ambiente online.

\footnotetext{
${ }^{4}$ Disponível em: https://drive.google.com/open?id=1hRAhu6iFJXwzImt4-cvsxUN2St3pl4us
} 
VIII Congresso Brasileiro de Informática na Educação (CBIE 2019)

Anais do XXX Simpósio Brasileiro de Informática na Educação (SBIE 2019)

Desta forma, é necessário repensar a estrutura colaborativa dos ambientes massivos, tendo em vista que o mesmo é visto como um repositório de materiais. Se o conteúdo for entregue de maneira monótona sem tirar vantagem de abordagens tecnológicas e didáticas avançadas, será difícil para os usuários permanecerem engajados. Consequentemente, um dos principais desafios é superar o problema de ser atraente para o público em massa e, ao mesmo tempo, tem que ser relevante para uma grande variedade de necessidades, requisitos e expectativas.

Uma alternativa para mudar este quadro detectado no estudo de caso seria a elaboração de um modelo de colaboração personalizado de acordo com as necessidades, perfis e habilidades dos alunos inscritos, visando a promoção da colaboração que é relevante para promoção da aprendizagem em MOOC, através da inserção de novas ferramentas de comunicação, bem como da possibilidade de criar um canal mais personalizado entre os alunos de acordo com o seu engajamento e habilidades.

Como trabalhos futuros, sugere-se realizar um diagnóstico dos padrões de comportamentos dos alunos através de Learning Analytics para agrupar os diversos alunos em categorias de acordo com o comportamento dos alunos no ambiente.

\section{REFERÊNCIAS}

ANTONACI, A.; KLEMKE, R.; STRACKE, C.M.; SPECHT, M. Gamification in MOOCs to enhance users' goal achievement. In Proceedings of the IEEE Global Engineering Education Conference (EDUCON 2017), Athens, Greece, 25-28 April 2017.

DOWNES, S. The Rise of Moocs, Knowledge, Learning, Community. 2012. Disponível em http://www.downes.ca/post/57911.

HOLANDA, A. C.; TEDESCO, P. MOOCs e Colaboração: definição, desafios, tendências e perspectivas. In: Anais do XXVIII Simpósio Brasileiro de Informática na Educação (SBIE). p. 243-252. 2017.

LERÍS, Dolores; SEIN-ECHALUCE; Hernandez, Miguel; BUENO, Concepción. Validation of indicators for implementing an adaptive platform for MOOCs. Published in: Journal Computers in Human Behavior. 2016. SCIENCE.

PIMENTEL, Mariano.; FUKS, Hugo. Sistemas Colaborativos. Ed. Elsevier. 2011.

STEIN, K. Penn GSE study shows MOOCs have relatively few active users, with only a few persisting to course end. Penn GSE Newsroom. 2013. Disponível em: https://www.gse.upenn.edu/news/press-releases/penn-gse-study-showsmoocs- haverelatively-few-active-users-only-few-persisting-

WAKS, Leonard J. The Evolution and Evaluation of Massive Open Online Courses MOOC In Motion. The Cultural and Social Foundations of Education. Temple University. Philadelphia, Pennsylvania, USA. 2016.

ZHENG, Saijing; WISNIEWSKI, Pamela; ROSSON, Mary Beth; CARROLl, John M. Ask the Instructors: Motivations and Challenges of Teaching Massive Open Online Courses. CSCW '16 Proceedings of the 19th ACM Conference on Computer-Supported Cooperative Work \& Social Computing. Page 206-221. 2016. 